\title{
Cooperative Effects of Corticosteroids and Catecholamines upon Immune Deviation of the Type- 1/Type-2 Cytokine Balance in Favor of Type-2 Expression in Human Peripheral Blood Mononuclear Cells
}

\author{
A.N. Salicrú, ${ }^{1}$ C.F. Sams, ${ }^{2}$ G.D. Marshall ${ }^{3}$
}

1 Department of Pathology and Laboratory Medicine, University of Texas Health Science Center at Houston 2 NASA Adaptations and Human Countermeasures Office

3 Division of Clinical Immunology and Allergy, University of Mississippi Medical Center

\begin{abstract}
A growing number of studies show strong associations between stress and altered immune function. In vivo studies of chronic and acute stress have demonstrated that cognitive stressors are strongly correlated with high levels of catecholamines (CT) and corticosteroids (CS). Although both CS and CT individually can inhibit the production of T-helper 1 (TH1, type-1 like) cytokines and simultaneously promote the production of Thelper 2 (TH2, type-2 like) cytokines in antigen-specific and mitogen stimulated human leukocyte cultures in vitro, little attention has been focused on the effects of combination $\mathrm{CT}$ and $\mathrm{CS}$ in immune responses that may be more physiologically relevant. We therefore investigated the combined effects of in vitro CT and CS upon the type-1/type-2 cytokine balance of human peripheral blood mononuclear cells (PBMC) as a model to study the immunomodulatory effects of superimposed acute and chronic stress. Results demonstrated a significant decrease in type-1 cytokine production (IFN- $\gamma$ ) and a significant increase in type-2 cytokine production (IL-4, IL-10) in our CS+CT incubated cultures when compared to either CT or CS agents alone. Furthermore, variable enhancement of type-1/type-2 immune deviation occurred depending upon when the CT was added. The data suggest that CS can increase the sensitivity of PBMC to the immunomodulatory effects of CT and establishes an in vitro model to study the combined effects of in vivo type-1/type-2 cytokine alterations observed in acute and chronic stress.
\end{abstract}




\section{Introduction}

A growing body of literature recognizes the dynamic interactions between components of the nervous, endocrine, and immune systems as a cohesive unit responsible for protection of the host ${ }^{(1-4)}$. In this model, products of the hypothalamic-pituitary-adrenal axis (HPA) and the sympathetic nervous system (SNS) shift immunity from an intracellular pathogen-specific (TH1, type-1 like) immune response to an extracellular pathogenspecific (TH2, type-2 like) response during times of high psychological stress $(1,4)$. Although this model of immune deviation is an adaptive mechanism that protects the host from the sequelae of systemic inflammation ${ }^{(3)}$, maladaptive responses to stress-induced immune deviation may exacerbate and/or contribute to the pathogenesis of immune-based clinical disorders ${ }^{(5)}$.

In vivo studies of chronic and acute stress situations such as those modeled by the Trier Social Stress Test (TSST) and seen in the medical student exam stress model (MSESM) have demonstrated that cognitive stressors such as public speaking and medical student exams are strongly correlated with high levels of CT and/or CS ${ }^{(6,7)}$. In addition, stressful events (particularly chronic) have been associated with adverse clinical effects such as viral reactivation and exacerbation of inflammatory disorders ${ }^{(7,8)}$. It seems reasonable to speculate that acutely stressful events might have an even more adverse clinical effect in individuals already chronically stressed, but this has not been well documented.

It has long been suggested that these effects can be partially explained by alterations of the cytokine network responsible for an optimally protective adaptive immune response (7). Studies by our group and others have shown that products of the HPA and SNS, namely CS and the CT epinephrine (EPI) and norepinephrine (NE) can alter the balanced expression of cytokines from $\mathrm{TH} 1$ in favor of $\mathrm{TH} 2$ in vitro ${ }^{(9-12)}$. Specifically, dexamethasone (DEX, a corticosteroid analog for cortisol used for its stable in vitro properties), EPI, and/or NE added as single agents at the initiation of cultures can alter the cytokine network responsible for the adaptive immune response by inhibiting the in vitro production of type-1 cytokines (IFN- $\gamma$, IL-12) while favoring the production of type-2 cytokines (IL-4, IL-10, IL-13) ${ }^{(10-12)}$.

While studies have largely investigated the individual effects of in vitro CS or CT in immune deviation, little attention has been focused upon the in vitro effects of combination CT and CS in immune responses that may be more physiologically relevant to the TRIER and medical student exam stress models. For example, CS can influence the long-term responses of various tissues to $\mathrm{CT}$ by increasing $\beta$-adrenergic receptor surface $(\beta 2-A R)$ expression and intracellular cAMP activity in human smooth muscle cells ${ }^{(13)}$ and epithelial cells ${ }^{(14,15)}$ lining the respiratory tract. More recent data suggest that $\beta 2$-AR expression on lymphocytes and APC may respond similarly to the long-term modulation of $\mathrm{CS}$ in murine and equine models ${ }^{(16-18)}$. In vitro exposure to CS can increase $\beta 2-\mathrm{AR}$ expression and cAMP activity in murine lymphocytes in vitro ${ }^{(16)}$ and murine and equine lymphocytes in vivo ${ }^{(17,18)}$. Furthermore, the combination of CT and CS decreased the pro-inflammatory cytokines IL-8, IL-6, and TNF- $\alpha$ in vitro from synovial tissue isolated in patients with rheumatoid arthritis ${ }^{(19)}$. Although documented, the combined effects of endogenous CS and CT on the type-1/type-2 cytokine network in human leukocytes are limited in the literature and have not been well studied. 
We therefore studied the combined physiological stress equivalents of endogenous CS and $\mathrm{CT}$ on antigen-specific stimulated human $\mathrm{PBMC}$ as an in vitro model of the immunomodulatory effects of superimposed chronic and acute stressors (i.e., neuroendocrine stress hormones). Our studies demonstrate that the combined effects of in-vitro CS and CT significantly decreases the production of type-1 cytokines and simultaneously increases the expression of type-2 cytokines in our antigen-specific stimulated PBMC cultures when compared to either CS or CT agents alone. These effects further shifted the balanced production of type-1/type-2 cytokines in favor of a type- 2 response. 


\section{Materials and Methods}

Reagents: EPI, NE, phorbol myristate acetate (PMA), ionomycin, and DEX were purchased from Sigma Chemicals (St. Louis, MO). Recombinant rhIL-2 was a gift from Dr. Jeffrey Rossio at the NIH-Frederick Cancer Research Center (Bethesda, MD). Tetanus toxoid (TT) was purchased from Massachusetts Biologic Laboratories (Boston, MA). ELISA kits are from Pharmingen (San Diego, CA).

Subjects: Fourteen healthy study subjects (evenly distributed between men and women) aged 18-50 were recruited for participation in the study (blood draws). Subjects were excluded for any past medical history of smoking, psychiatric illnesses, cardiovascular diseases, or recent medical use of CS, CT, and adrenergic antagonists. Blood samples were drawn in the morning between 0800 and 1200 hours to control for diurnal variation in immune responses. The Committee for the Protection of Human Subjects (CPHS) at the University of Texas Medical School at Houston approved protocols for the study. Informed consent was obtained from each subject prior to enrollment.

Isolation of PBMC: Venous blood was collected in heparinized Vacutainer tubes (Becton Dickinson, San Diego, CA) and PBMC were isolated via ficoll-hypaque density gradient centrifugation at $3000 \mathrm{~g}$ for 20 minutes. Cells were washed twice with $\mathrm{Ca} 2+/ \mathrm{Mg} 2+$ free HBSS (Sigma), and resuspended in RPMI 1640 supplemented with 90U/ml penicillin, $90 \mu \mathrm{g} / \mathrm{ml}$ streptomycin, $2 \mathrm{mMol} / \mathrm{L} \mathrm{L-Glutamine} \mathrm{(all} \mathrm{from} \mathrm{Sigma),} \mathrm{and}$ $10 \%$ human AB serum (Atlanta Biologicals).

PBMC Cultures: For incubation experiments, DEX at concentrations of $1 \times 10^{-8} \mathrm{M}$ (mimicking the stress-physiologic plasma levels ${ }^{(11)}$ of endogenous glucocorticoids) was added on the first day of stimulation followed by the addition of EPI or NE at the stress physiologic concentrations $\left(1 \times 10^{-8} \mathrm{M}, 1 \times 10^{-7} \mathrm{M}\right.$, respectively) on fifth and tenth days of stimulation. PBMC Cultures at $1 \times 10^{6}$ cells $/ \mathrm{ml}$ were stimulated with $0.5 \mu \mathrm{g} / \mathrm{ml}$ TT and 10 $\mathrm{IU} / \mathrm{ml}$ rhIL-2 for 5 days at $37^{\circ} \mathrm{C}$ and $5 \%$ carbon dioxide for all experiments as previously described $(11,12)$. On day $5,0.5 \mathrm{ml}$ of supernatant was removed and replaced with 20 $\mathrm{IU} / \mathrm{ml} \mathrm{rhIL}-2$ for cellular expansion and $0.5 \mathrm{ml}$ fresh media containing $10 \%$ human $\mathrm{AB}$ serum. On day $10,0.5 \mathrm{ml}$ of supernatant was removed and replaced with $0.5 \mathrm{ml}$ media containing $10 \mu \mathrm{g} / \mathrm{ml}$ phytohemagluttinin (PHA) and $1 \mathrm{ng} / \mathrm{ml}$ phorbol myrisitate acetate (PMA) and incubated for 24 hours as previously described ${ }^{(11,12)}$. Briefly, a clonal population of antigen specific T-lymphocytes predominates by the end of the 11 day culture period. These clonal cells are stimulated with PHA and PMA on the $10^{\text {th }}$ day of culture for 24 hours to amplify the production of cytokines from phenotypically committed cells that otherwise produce cytokines at levels too low for detection by ELISA. The use of mixed-cell cultures such as PBMC is closer to physiological conditions than cultures using isolated CD4+ naïve T-cells for our set of experiments. For cultures coincubated with both CS and CT (simulating acute stress episodes superimposed upon chronic stress), EPI was added for convenience on the $5^{\text {th }}$ and $10^{\text {th }}$ days at the same time the media was scheduled for change as previously described ${ }^{(11,12)}$. Supernatants were harvested on day 11 and stored at $-70^{\circ} \mathrm{C}$.

Cytokine analysis: Levels of type- 1 and type- 2 cytokines in culture supernatants were determined by enzyme linked immunosorbent assays (ELISA) using paired antibodies specific for IFN- $\gamma$, IL-10, and IL-4 (Pharmingen). Developed ELISA plates were read on an Emax plate reader (Molecular Devices) at 450nm. Sample concentrations were determined based on calculated standard curves using SOFTmax (Molecular Devices). The lower limit of detection of the IFN- $\gamma$, IL-10, and IL-4 kits was $4 \mathrm{pg} / \mathrm{ml}$.

Statistical methods: Cytokine data (with the exception of Type-1/Type-2 ratio data) were transformed into a logarithmic scale $\left(\log _{10}\right)$ to normalize the results, thus bringing 
the data closer to a Gaussian distribution. Data are presented in graph form as mean \pm standard deviation (SD). Type-1/Type-2 cytokine ratios were calculated from untransformed cytokine values for each individual and culture condition before summarized in bar graph format. The data are presented as mean \pm SD. Comparisons between 3 or more groups utilized the repeated measures one-way analysis of variance (ANOVA) with post test analysis for group differences by Tukey's Multiple Comparisons Test. Statistical analyses were calculated with Prism (Version 4.0 Graphpad Software, San Diego, CA).

\section{Results}

\section{Decreased Production of Type-1 Cytokines by Combined Physiological Stress Levels of Corticosteroids and Catecholamines}

To determine the combined effects of adrenergic agents and CS, we used physiological stress concentrations of EPI ${ }^{(12)}$ and DEX (mimicking the stress-physiologic plasma levels of endogenous glucocorticoids) ${ }^{(1)}$ together or as single agents. In these sets of experiments IFN- $\gamma$ levels were determined in culture supernatants from TT stimulated cultures treated with combination DEX at $10^{-8} \mathrm{M}$ and EPI at $10^{-8} \mathrm{M}$. Coincubation cultures with DEX and EPI were added either on day one of stimulation (DEX+EPI $)$ or DEX on the $1^{\text {st }}$ day followed by EPI added on $5^{\text {th }}$ and $10^{\text {th }}$ days of antigenic stimulation $\left(\mathrm{DEX}+\mathrm{EPI}_{5,10}\right)$. In addition, $\mathrm{DEX}, \mathrm{EPI}_{1}$ (added as a single agent on the first day of stimulation) and $\mathrm{EPI}_{5,10}$ (EPI added as a single agent on the $5^{\text {th }}$ and $10^{\text {th }}$ days of stimulation) groups were added as supplementary controls. The means from the treatment groups differed significantly by repeated measures ANOVA $(p<0.0001)$. Tukey's post-test analysis (TPTA) revealed significant inhibition of IFN- $\gamma$ production from baseline control in the $\mathrm{DEX}, \mathrm{EPI}_{1}, \mathrm{DEX}+\mathrm{EPI}_{1}, \mathrm{DEX}+\mathrm{EPI}_{5,10}$ groups. In addition, TPTA demonstrated significantly more inhibition of IFN- $\gamma$ production from both the $\mathrm{DEX}$ and $\mathrm{DEX}+\mathrm{EPI}_{1}$ groups. The mean of the $\mathrm{DEX}+\mathrm{EPI}_{1}$ treated group did not differ significantly from the DEX treated group, suggesting that the inhibition of IFN- $\gamma$ in the $\mathrm{DEX}+\mathrm{EPI}_{1}$ group was due to DEX and not to the additive effect of EPI in long-term antigen-specific stimulated cultures (Figure 1).

\section{Increased Production of Type-2 Cytokines by Combined Stress Levels of Corticosteroids and Catecholamines}

Production of IL-10 and IL-4 production was determined from the same culture supernatants described above. The means for both IL-4 and IL-10 among the treatment groups differed significantly by repeated measures ANOVA $(\mathrm{p}<0.0001)$. TPTA revealed a significant increase of IL-10 production in the DEX, EPI $1, \mathrm{DEX}+\mathrm{EPI} \mathrm{I}_{1}$, and $\mathrm{DEX}+\mathrm{EPI}_{5,10}$ groups from baseline control (Figure 2). In addition, TPTA revealed a significant increase in IL-4 production in the $\mathrm{EPI}_{1}$ and $\mathrm{DEX}+\mathrm{EPI}_{5,10}$ groups but not the $\mathrm{DEX}, \mathrm{EPI}_{5,10}$, or $\mathrm{DEX}+\mathrm{EPI}_{1}$ groups when compared to control (Figure 3). Furthermore, TPTA revealed that the DEX+EPI ${ }_{5,10}$ group significantly increased the production of IL-4 and IL-10 over the DEX, DEX+EPI ${ }_{1}$, and $\mathrm{EPI}_{1}$ groups (Figures 2, 3).

A balanced production of cytokines is needed for an adaptive immune response that is appropriate for the inciting antigen ${ }^{(20)}$. To further investigate the impact of CS and CT on this balance, type-1/type- 2 cytokine ratios were calculated based upon cytokine expression levels for all treatment groups demonstrated in Figures 1-3. The group means 
in the IFN- $\gamma /$ IL-10 graph (Figure 4) and IFN- $\gamma /$ IL-4 (Figure 5) differed significantly by repeated measures ANOVA $(\mathrm{p}<0.0001)$. The IFN- $\gamma /$ IL10 ratios were significantly decreased in the DEX, $\mathrm{EPI}_{1}, \mathrm{DEX}+\mathrm{EPI}_{1}$, and $\mathrm{DEX}+\mathrm{EPI}_{5,10}$ groups when compared to baseline control from TPTA (Figure 4). Similarly, the IFN- $\gamma /$ IL-4 ratios were significantly decreased in the DEX, $\mathrm{EPI}_{1}, \mathrm{DEX}+\mathrm{EPI}_{1}$, and $\mathrm{DEX}+\mathrm{EPI}_{5,10}$ groups from baseline control (Figure 5) by TPSA. The type-1/type-2 ratio in the DEX+EPI 5 group was significantly less than the DEX, EPI ${ }_{1}$, and DEX+EPI ${ }_{1}$ by TPTA In both the type1/type-2 graphs (Figures 4 and 5), demonstrating that the additive effects of EPI added several days later to long-term antigen-specific cultures significantly decreased the balanced production of cytokines further in favor of type-2 cytokines.

\section{Propanolol Blocks the Type-2 Cytokine Shift from the Additive Effects of $\beta 2$ - Agonists}

We wished to investigate if the cooperative effects of CS and CT were mediated in part by the $\beta 2$-adrenergic receptor ( $\beta 2$-AR). Previously, PRO, a non-selective $\beta$-adrenergic receptor antagonist, has been shown to mitigate the in vitro effects of catecholamines in antigen-specific PBMC cultures (X). The mean production of IFN- $\gamma$, IL-10, and IL-4 in the DEX $+\mathrm{EPI}_{5,10}+\mathrm{PRO}$ group did not significantly differ from the DEX group (Figures 1 - 3) by TPTA. However, the mean production of IFN- $\gamma$ in the DEX+EPI ${ }_{5,10}$ group was significantly less than the $\mathrm{DEX}+\mathrm{EPI}_{5,10}+\mathrm{PRO}$ group by TPTA. In addition, the mean production of type-2 cytokines (IL-10 and IL-4) were significantly higher in the $\mathrm{DEX}+\mathrm{EPI}_{5,10}$ group than the $\mathrm{DEX}+\mathrm{EPI}_{5,10}+\mathrm{PRO}$ group by TPTA suggesting that the observed differences were mediated by $\beta$-agonists (Figures $1-3$ ). In addition, the type1/type-2 ratios between the DEX+EPI $5,10+\mathrm{PRO}$ and the DEX groups (Figure 4,5 ) did not significantly differ by TPTA suggesting that the significant differences between the $\mathrm{DEX}+\mathrm{EPI}_{5,10}$ and DEX groups were mediated by $\beta$-agonists (Figures 4,5 ). 


\section{Discussion}

Recent studies have shown that CT amplify the anti-inflammatory effects of CS in fibroblasts and smooth muscle cells ${ }^{(21-24)}$, thus attenuating the effects of CT-induced tachyphylaxis of the $\beta 2-\mathrm{AR}$ ( $\beta 2-\mathrm{AR}$ desensitization) in human respiratory smooth muscle cells $(22,25)$. In addition, the combination of $\mathrm{CT}$ and $\mathrm{CS}$ has a cooperative antiinflammatory effect on synovial tissue by inhibiting type-1 cytokines in vitro and in vivo (19). Furthermore, the combined effects of pharmacologically available adrenergic agonists with CS suppress T-cell proliferation and type-1 cytokine production in shortterm mitogen-stimulated human PBMC cultures in vitro, although variable effects were observed with type-2 cytokines ${ }^{(26)}$. However, these observations do not address physiologic parameters such as the use of stress doses of endogenous CT with CS on antigen-specific immune responses that would model in vivo stress-induced immune dysfunction.

The goal of the study was to develop an in vitro antigen-specific model to examine the immune effects of endogenous CT stress hormones that appear acutely in the context of chronic stress CS hormones. These experiments demonstrated that the combination of CS and CT significantly decreased the production of IFN- $\gamma$ and simultaneously increased the production of IL-10 and IL-4 when compared to either agent alone (Figures 1-5). Furthermore, the balanced production of type-1/type- 2 cytokines significantly shifted towards a type- 2 cytokine profile in the $\mathrm{DEX}+\mathrm{EPI}_{5,10}$ group over the $\mathrm{DEX}+\mathrm{EPI}_{1}, \mathrm{DEX}$, and $\mathrm{EPI}_{1}$ groups (figures 4,5). The EPI-mediated cytokine alterations were blocked by PRO indicating that the cooperative effects were mediated through the $\beta 2-\mathrm{AR}$. Our results are novel in that both type- 2 cytokines in our study (IL-4 and IL-10) were significantly increased and IFN- $\gamma$ was significantly decreased using long-term antigenspecific stimulated human PBMC cultures. In addition, these results were obtained using physiologic stress concentrations of endogenous CT (EPI, NE) in contrast to therapeutic doses of pharmacologically available long-acting $\beta$-agonists in short-term mitogenstimulated cultures.

Of note, we observed variations in cytokine expression levels from different study subjects. For example, the $\mathrm{DEX}+\mathrm{EPI}_{5,10}$ group had significantly lower type-1 cytokine production and increased type-2 cytokines, although some individuals were more sensitive than others to the cooperative effects of CS and CT. These within group differences may be at least partially explained by subject heterogeneity such as functional polymorphisms of the $\beta 2-\mathrm{AR}^{(27-29)}$, glucocorticoid receptor ${ }^{(30,31)}$, and cytokine receptors $(32,33)$. In addition to polymorphisms, different isoforms of the GCR due to transcript alternative splicing and alternative translation initiation ${ }^{(34-36)}$ may also contribute to variation of cytokine expression and potentially immune deviation of the type-1/type- 2 cytokine balance. These possibilities await future investigation.

Kinetic differences were observed between our $\mathrm{DEX}+\mathrm{EPI}_{1}$ and $\mathrm{DEX}+\mathrm{EPI}_{5,10}$ cultures (Figures 1-5). In particular, these two treatment groups demonstrated that the cooperative effects of combination CS and endogenous CT in antigen-specific cultures appear at later time points $\left(\mathrm{DEX}+\mathrm{EPI}_{5,10}\right.$ cultures) rather than earlier exposure to adrenergic agents $\left(\mathrm{DEX}+\mathrm{EPI}_{1}\right.$ cultures) in our antigen-specific cultures. Combined with the adrenergic 
antagonist data with $\mathrm{PRO}$, the data suggest that the mechanism responsible for these observations is time-dependent and may therefore require modification or de novo synthesis of the AR or CS signaling apparatus after antigenic-stimulation. Possible mechanisms may include increased nuclear translocation of the GCRC ${ }^{(26)}$, increased cell surface AR density and function (from de novo synthesis or redistribution of internalized receptors between the internal and external cellular compartments), and/or increased secondary signaling from the AR.

Although our DEX $+\mathrm{EPI}_{1}$ data differed from those previously reported by Goleva et al ${ }^{(26)}$, several differences in the experimental design may explain these discrepancies. Goleva et al used short-term mitogen stimulation (PMA and Ionomycin) vs. long-term antigenspecific cultures (TT) used in our study. Mitogen stimulation requires shorter incubation periods than antigen-specific stimulation due to bypassing antigen presentation, costimulation by the B7 receptor family (i.e., B7.1, B7.2), and differentiation of naïve Tcells (TH0) into TH1 and TH2 clones. Moreover, the physical and biological differences between endogenous $\mathrm{CT}$ and long-acting synthetic $\beta$-agonists such as salmeterol include elimination half-lives of 2-3 minutes for EPI compared to 5.5 hours ${ }^{(37,38)}$ for salmeterol due in part to the lipophilic nature of salmeterol, reduced ability of salmeterol to induce tachyphylaxis of $\beta 2-\mathrm{AR}^{(38)}$, alternate routes of metabolism, and the relative instability of endogenous CT (EPI and NE) at culture $\mathrm{pH}$ and temperature. In effect, these differences may allow for the in vitro manifestations of long-acting $\beta$-agonists on PBMC to appear more readily in short term cultures than the additive effects of EPI or NE manifested in long-term antigen-specific cultures until later time points.

Based upon preliminary experiments, the likely sources of expressed cytokines in our long-term antigen-specific cultures were clonal populations of tetanus-specific CD4+ Tlymphocytes. Greater than $90 \%$ of the viable cells remaining in culture at day 11 were CD4+ T-lymphocytes, 5-9\% were CD8+ T-lymphocytes, and less than 1\% were CD19+ B-lymphocytes (data not shown). CD14+ APC, also a source of IL-10, were not detected. This suggests that immune deviation of the type-1/type- 2 paradigm had occurred primarily at the level of the T-cell in our in vitro model.

In summary, our study was designed to establish an in vitro human model of stress hormone-induced immune changes. This model demonstrated that the combination of CS and CT enhanced immune deviation of the cytokine network towards a type-2 adaptive immune response to recall antigen (TT) in vitro. Future research will explore possible mechanisms for the cooperative effects of combination CS and CT on immune function such as changes in the function and expression of $\beta 2-\mathrm{AR}$ on specific leukocyte populations (lymphocytes and APC) as a complementary mechanism to increased nuclear translocation of the GCRC. Additionally, our in vitro model can serve as a guide to developing future clinical studies that will investigate specific immune dysfunction after acutely stressful situations in patients who are already chronically stressed. Understanding these effects should allow development of interventional strategies to mitigate or prevent the adverse immunological effects of acute and chronic stress in patients with various inflammatory diseases. 


\section{References}

1. Elenkov, I., Chrousos, G. 2002. Stress hormones, proinflammatory and antiinflammatory cytokines, and autoimmunity. Ann of the New York Academy of Sciences. 966: 290-303

2. Chrousos, G.P. 1995. The hypothalamic-pituitary-adrenal axis and immunemediated inflammation. New England Journal of Medicine. 332: 1351-1362

3. Webster, J., Tonelli, L., Sternberg, E.M. 2002. Neuroendocrine Regulation of Immunity. Ann. Reviews in Immunology. 20: 125-163

4. Sanders, V.M., Straub, R.H. 2002. Norepinephrine, the $\beta$-adrenergic receptor, and immunity. Brain, Behavior, and Immunity. 16: 290-332

5. Glaser, R., Kiecolt-Glaser, J.K. 2005. Stress-induced immune dysfunction: implications for health. Nature Reviews. Immunology. 5: 243-251

6. Kirschbaum, C., Pirke, K.M., Hellhammer, D.H. 1993. "The Trier Social Stress Test": A tool for investigating psychobiological stress responses in a laboratory setting. Neuropsychobiology. 28: 76-81

7. Marshall, G.D. Jr., Agarwal, S.K., Lloyd, C., Cohen, L., Henninger, E.M., Morris, G.J. 1998. Cytokine dysregulation associated with exam stress in healthy medical students. Brain Behavior and Immunity. 12: 297-307

8. Buske-Kirschbaum, A., Gierens, A., Hollig, H., Hellhammer, D.H. 2002. Stress induced immunomodulation is altered in patients with atopic dermatitis. Journal of Neuroimmunology. 129: 161-167

9. Schwiebert, L.M., Beck, L.A., Stellato, C., Bickel, C.A., Bochner, B.S., Schleimer, R.P. 1996. Glucocorticosteroid inhibition of cytokine production: relevance to anti-allergic actions. Journal of Allergy and Clinical Immunology. 97: 143-152.

10. Elenkov, Ilia J., Wilder, R.L., Chrousos, G.P., Vizi, E.S. 2000. The sympathetic nerve - an integrative interface between two supersystems: the brain and the immune system. Pharmacological Reviews. 52: 595-638

11. Agarwal, S., Marshall, G.D. 1998. Glucocorticoid induced type-1/type-2 alterations in humans: a model for stress related immune dysfunction. Journal of Interferon and Cytokine Research. 18: 1059-1068

12. Agarwal, S.K., Marshall, G.D., 2000. $\beta$-Adrenergic Modulation of Human Type1/Type-2 Cytokine Balance. Journal of Allergy and Clinical Immunology. 105: 91-98

13. Kalavantavanich, K., Schramm, C.M. 2000. Dexamethasone potentiates highaffinity beta-agonist binding and $\mathrm{g}(\mathrm{s})$ alpha protein expression in airway smooth muscle. American Journal of Physiology. Lung Cellular and Molecular Physiology. 278: 1101-1106

14. Aksoy, M.O., Mardini, I.A., Yang, Y., Bin, W., Zhou, S., Kelson, S.G. 2002. Glucocorticoid effects on the beta-adrenergic receptor-adenylyl cyclase system of human airway epithelium. Journal of Allergy and Clinical Immunology. 109: 491-497

15. Mak, J.C., Nishikawa, M., Shirasaki, H., Miyayasu, K., Barnes, P.J. 1995. Protective effects of a glucocorticoid on downregulation of pulmonary beta 2adrenergic receptors in vivo. Journal of Clinical Investigation. 96: 99-106 
16. Baus, E., Van Laethem, F., Andris, F., Rolin, S., Urbain, J., Leo, O. 2001. Dexamethasone increases intracellular cyclic AMP concentration in murine $\mathrm{T}$ lymphocyte cell lines. Steroids. 66: 39-47

17. Edgar, V.A., Silberman, D.M., Cremaschi, G.A., Zieher, L.M., Genaro, A.M. 2003. Altered lymphocyte catecholamine reactivity in mice subjected to chronic mild stress. Biochemical Pharmacology. 65: 15-23

18. Abraham, G., Schusser, G.F., Ungemach, F.R. 2003. Dexamethasone-induced increase in lymphocyte beta-adrenergic receptor density and cAMP formation in vivo. Pharmacology. 67: 1-5

19. Straub, R.H., Gunzler, C., Miller, L.E., Cutolo, M., Scholmerich, J., Schill, S. 2002. Anti-inflammatory cooperativity of corticosteroids and norepinephrine in rheumatoid arthritis synovial tissue in vivo and in vitro. FASEB Journal. 16: 993-1000.

20. Imami, N., Pires, A., Hardy, G., Wilson, J., Gazzard, B., Gotch, F. 2002. A balanced type-1/type-2 response is associated with long-term nonprogressive human immunodeficiency virus type 1 infection. Journal of Virology. 76: 90119023

21. Johnson, M. 2004. Interactions between corticosteroids and beta2-agonists in asthma and chronic obstructive pulmonary disease. Proceedings of the American Thoracic Society. 1(3): 200-6

22. Tan, K.S., McFarlane, L.C., Lipworth, B.J. 1998. Concomitant administration of low-dose prednisolone protects against In Vivo $\beta 2$-Adrenoreceptor subsensitivity induced by regular formoterol. Chest. 113: 34-41

23. Eickelberg, O., Roth, M., Lorx, R., Bruce, V., Rudiger, J., Johnson, M., Block, L.H. 1999. Ligand-independent activation of the glucocorticoid receptor by the $\beta 2$-adrenergic receptor agonists in primary human lung fibroblasts and vascular smooth muscle cells. Journal of Biological Chemistry. 274:1005-1010

24. Adcock, I.M., Maneechotesuwan, K., Usmani, O. 2002. Molecular interactions between glucocorticoids and long-acting $\beta 2$-agonists. Journal of Allergy and Clinical Immunology. 110:S261-268

25. Sin, D.D., Johnson, M., Gan, W.Q., Paul Man, S.F. 2004. Combination therapy of inhaled corticosteroids and long-acting $\beta 2$-Adrenergics in management of patients with chronic obstructive pulmonary disease. Current Pharmaceutical Design. 10: 3547-3560

26. Goleva, E., Dunlap, A., Leung, D.Y. 2004. Differential control of TH1 versus TH2 cell responses by the combination of low-dose steroids with beta2-adrenergic agonists. Journal of Allergy and Clinical Immunology. 114:183-91

27. Brodde, O.E., Leineweber, K. 2005. Beta-2 adrenoceptor gene polymorphisms. Pharmacogenetics and Genomics. 15: 265-275

28. Masuo, K., Katsuya, T., Fu, Y., Rakugi, H., Ogihara, T., Tuck, M.L. Beta2adrenoceptor polymorphisms relate to insulin resistance and sympathetic overactivity as early markers of metabolic disease in non-obese, normotensive individuals. American Journal of Hypertension: Journal of the American Society of Hypertension. 18: 1009-1014 
29. Hancox, R.J., Sears, M.R., Taylor, D.R. 1998. Polymorphism of the $\beta 2-$ adrenoreceptor and the response to long-term beta2-agonist therapy in asthma. European Respiratory Journal. 11: 589-593

30. Russcher, H., Smit, P., van den Akker, E.L., van Rossu, E.F., Brinkmann, A.O., de Jong, F.H., Lamberts, S.W., Koper, J.W. 2005. Two polymorphisms in the glucocorticoid receptor gene directly affect glucocorticoid-regulated gene expression. Journal of Clinical Endocrinology and Metabolism. 90: 5804-5810

31. Bjorntorp, P. 2002. Alterations in the ageing corticotropic stress-response axis. Novartis Foundation Symposium. 242: 46-58

32. Kabesch, M., Schedel, M., Carr, D., Woitsch, B., Fritzsch, C., Weiland, S.K., von Mutius, E. 2006. IL-4/IL-13 pathway genetics strongly influence serum IgE levels and childhood asthma. Journal of Allergy and Clinical Immunology. 117: 269-274

33. Dean, M., Carrington, M., O’Brien, S.J. 2002. Balanced polymorphism selected by genetic versus infectious human disease. Annual Reviews in Genomics and Human Genetics. 3: 263-292

34. Lu, N.Z., Cidlowski, J.A. 2004. The origin and functions of multiple human glucocorticoid receptor isoforms. Annals of the New York Academy of Sciences. 1024: $102-123$

35. Pujols, L., Mullol, J., Roca-Ferrer, J., Torrego,A., Xaubet, A., Cidlowski, J.A., Picado, C. 2002. Expression of glucocorticoid receptor alpha- and beta-isoforms in human cells and tissues. American Journal of Physiology. Cell Physiology. 283: C1324-C1331

36. Hagendorf, A., Koper, J.W., de Jong, F.H., Brinkmann, A.O., Lamberts, S.W., Feelders, R.A. 2005. Expression of the human glucocorticoid receptor splice variants alpha, beta, and $\mathrm{P}$ in peripheral blood mononuclear leukocytes in healthy controls and in patients with hyper- and hypocortisolism. The Journal of Clinical Endocrinology and Metabolism. 90: 6237-6243

37. Lehtonen, L.A., Antila, S., Pentikäinen, P.J. 2004. Pharmacokinetics and pharmacodynamics of intravenous inotropic agents. Clinical Pharmacokinetics. 43: $187-203$

38. Cazzola, M., Testi, R., Matera, M.G. 2002. Clinical pharmacokinetics of salmeterol. Clinical Pharmacokinetics. 41: 19-30 
Figure 1

IFN- $\gamma$ Production

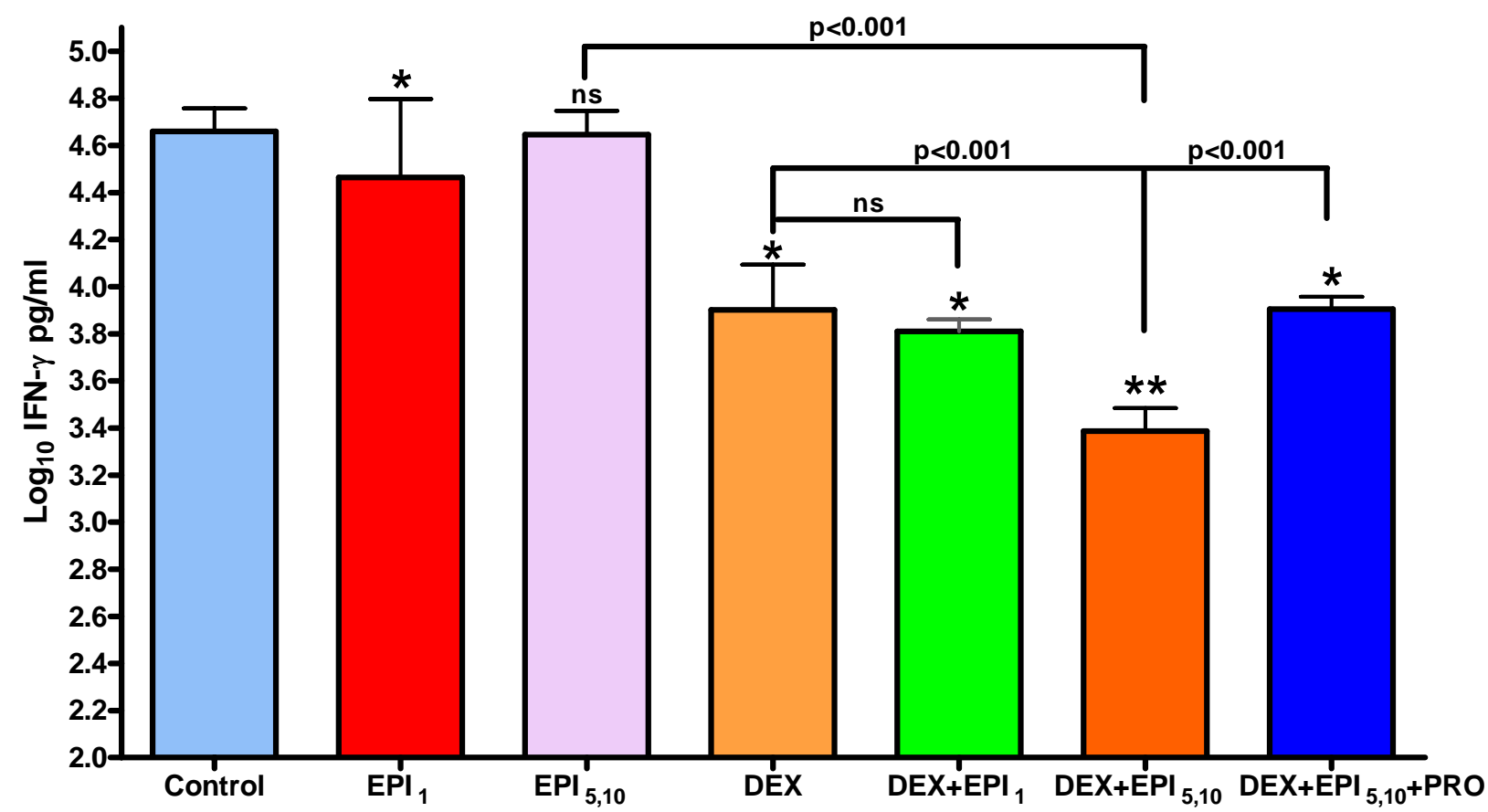

Legend For Figures 1 - 5

$\square \mathrm{EPI}_{1}=\mathrm{EPI}$ added on the 1 st day of culture

$\square \mathrm{EPI}_{5,10}=\mathrm{EPI}$ added on the 5th and 10th days of culture

DEX = DEX added on the 1st day of culture

$\square \mathrm{DEX}+\mathrm{EPI}_{1}=$ Combination of DEX and EPI added on the 1st day of culture

$\square$ DEX+EPI ${ }_{5,10}=$ Combination of DEX added on the 1st day of culture and

EPI subsequently added on the 5 th and 10th days of culture

$\square \mathrm{PRO}=$ Beta receptor blockade by propranolol

Figure 1: Detection of IFN- $\gamma$ production in 11-day Tetanus-Toxoid (TT) stimulated cultures by ELISA. The data represent the effects of physiologic-stress doses of $\mathrm{CT}$ added at different time points $\left(\mathrm{EPI}_{1}, \mathrm{EPI}_{5,10}\right), \mathrm{CS}(\mathrm{DEX})$, and the combination of CT+CS (DEX+EPI, $\mathrm{DEX}+\mathrm{EPI}_{5,10}$ ) in human PBMC from a sample size of 14 healthy adults. Data are represented as mean \pm standard deviation. $*=\mathbf{p}<0.5, * *=\mathbf{p}<0.001$, and represented $p$ values calculated by Tukey's Multiple Comparisons test. 
Figure 2

\section{IL-10 Production}

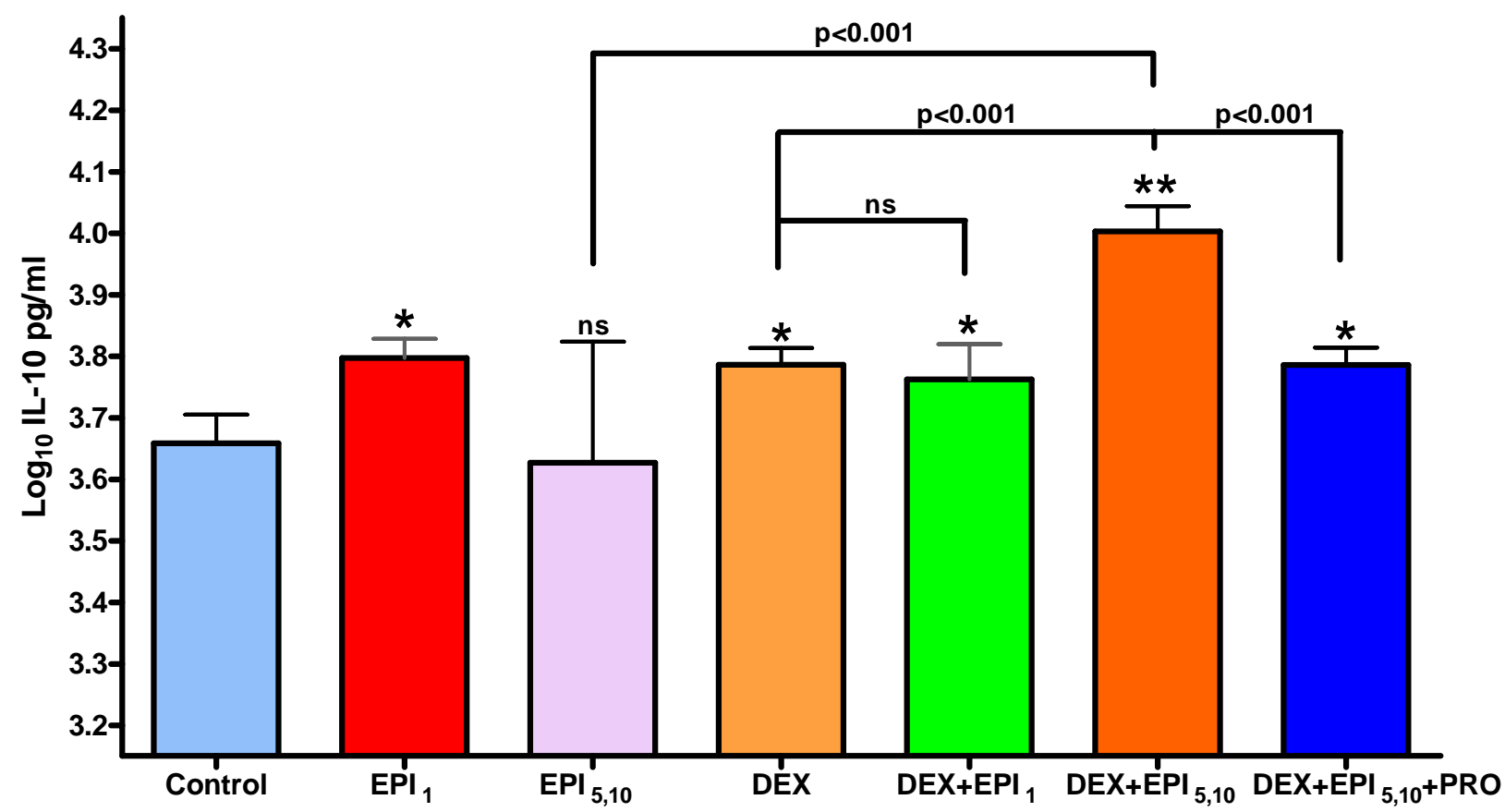

Figure 2: Detection of IL-10 production in 11-day TT (antigen-specific) stimulated cultures by ELISA. The data represent the effects of physiologic-stress doses of $\mathrm{CT}$ added at different time points $\left(\mathrm{EPI}_{1}, \mathrm{EPI}_{5,10}\right), \mathrm{CS}(\mathrm{DEX})$, and the combination of CT+CS (DEX+EPI, $\left.\mathrm{DEX}+\mathrm{EPI}_{5,10}\right)$ in human PBMC from a sample size of 14 healthy adults. Data are represented as mean \pm standard deviation. $*=\mathbf{p}<0.5, * *=\mathbf{p}<0.001$, and represented $p$ values calculated by Tukey's Multiple Comparisons test. 
Figure 3

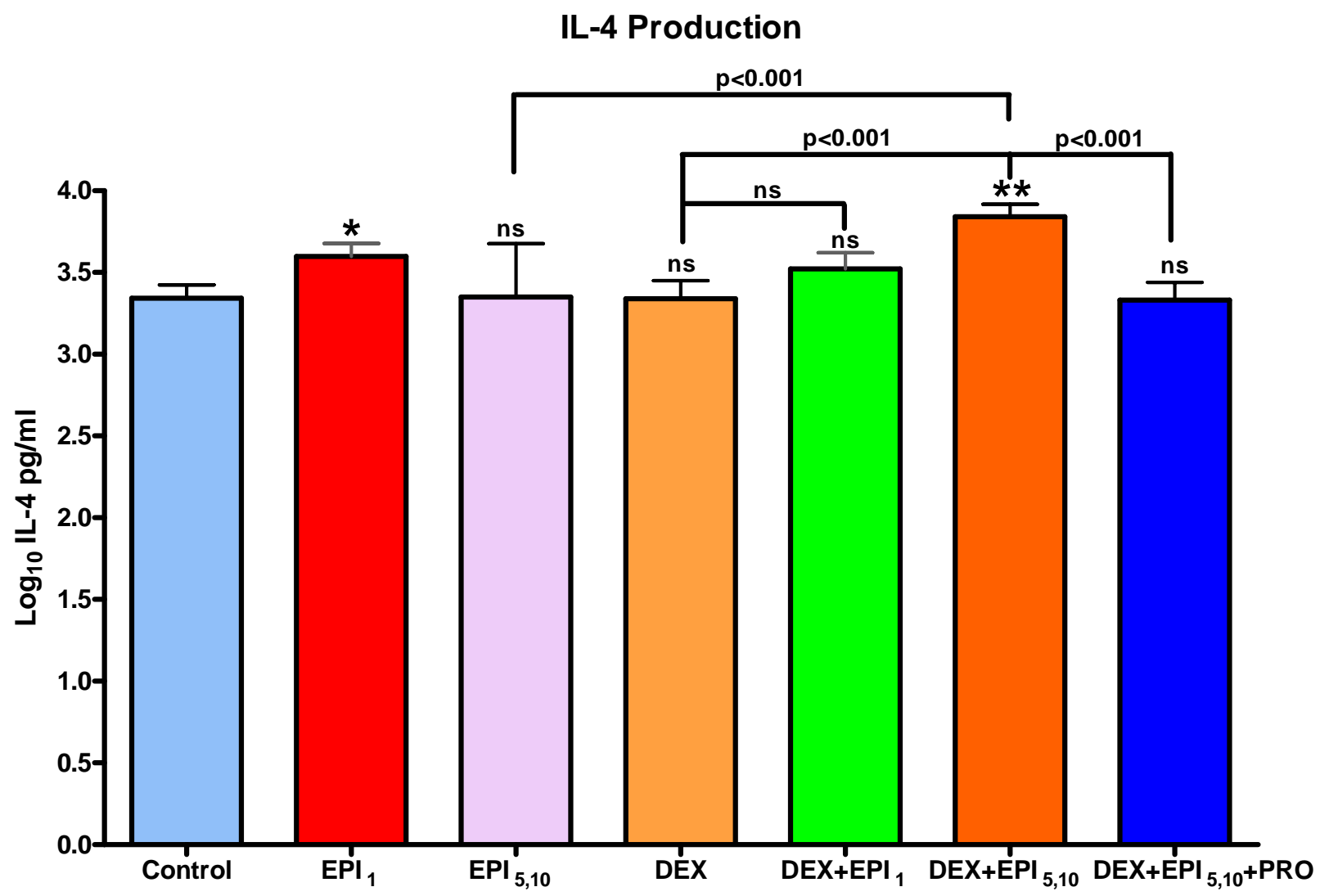

Figure 3: Detection of IL-4 production in 11-day TT (antigen-specific) stimulated cultures by ELISA. The data represent the effects of physiologic-stress doses of CT added at different time points (EPI, $\left.\mathrm{EPI}_{5,10}\right), \mathrm{CS}(\mathrm{DEX})$, and the combination of CT+CS (DEX+EPI, $\mathrm{DEX}+\mathrm{EPI}_{5,10}$ ) in human PBMC from a sample size of 14 healthy adults. Data are represented as mean \pm standard deviation. $*=\mathbf{p}<0.5, * *=\mathbf{p}<0.001$, and represented $p$ values calculated by Tukey's Multiple Comparisons test. 
Figure 4

Type-1/Type-2 Ratio

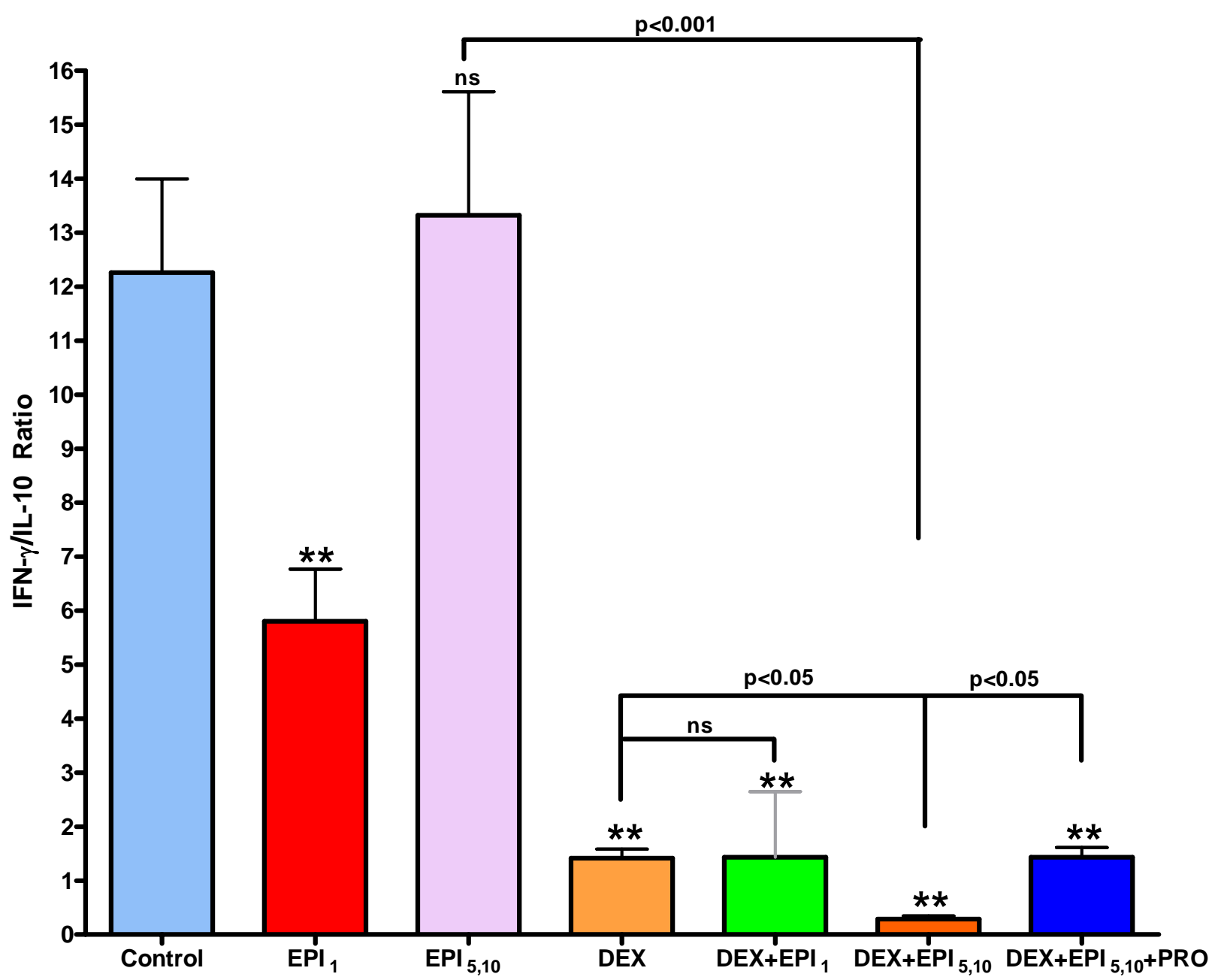

Figure 4: The balanced production of cytokines determines the adaptive immune response. Type-1/Type-2 (IFN- $\gamma /$ IL-10) cytokine ratios were calculated from ELISA data of 11-day TT (antigen-specific) stimulated cultures. The balanced production of cytokines determines the adaptive immune response. Type-1/Type-2 (IFN- $\gamma /$ IL-10) cytokine ratios were calculated from ELISA data of 11-day TT (antigen-specific) stimulated cultures. The data represent the effects of physiologic-stress doses of CT added at different time points (EPI, $\left.\mathrm{EPI}_{5,10}\right), \mathrm{CS}$ (DEX), the combination of CT+CS $\left(\mathrm{DEX}+\mathrm{EPI}_{1}, \mathrm{DEX}_{+} \mathrm{EPI}_{5,10}\right)$, or $\mathrm{CT}+\mathrm{CS}+$ propranolol $\left(\mathrm{DEX}+\mathbf{E P I}_{5,10}\right.$ + PRO) in human PBMC from a sample size of 14 healthy adults. Data are represented as mean \pm standard deviation. $* *=\mathbf{p}<0.001$ and represented $\mathbf{p}$ values were calculated by Tukey's Multiple Comparisons test. 
Figure 5

Type-1/Type-2 Ratio

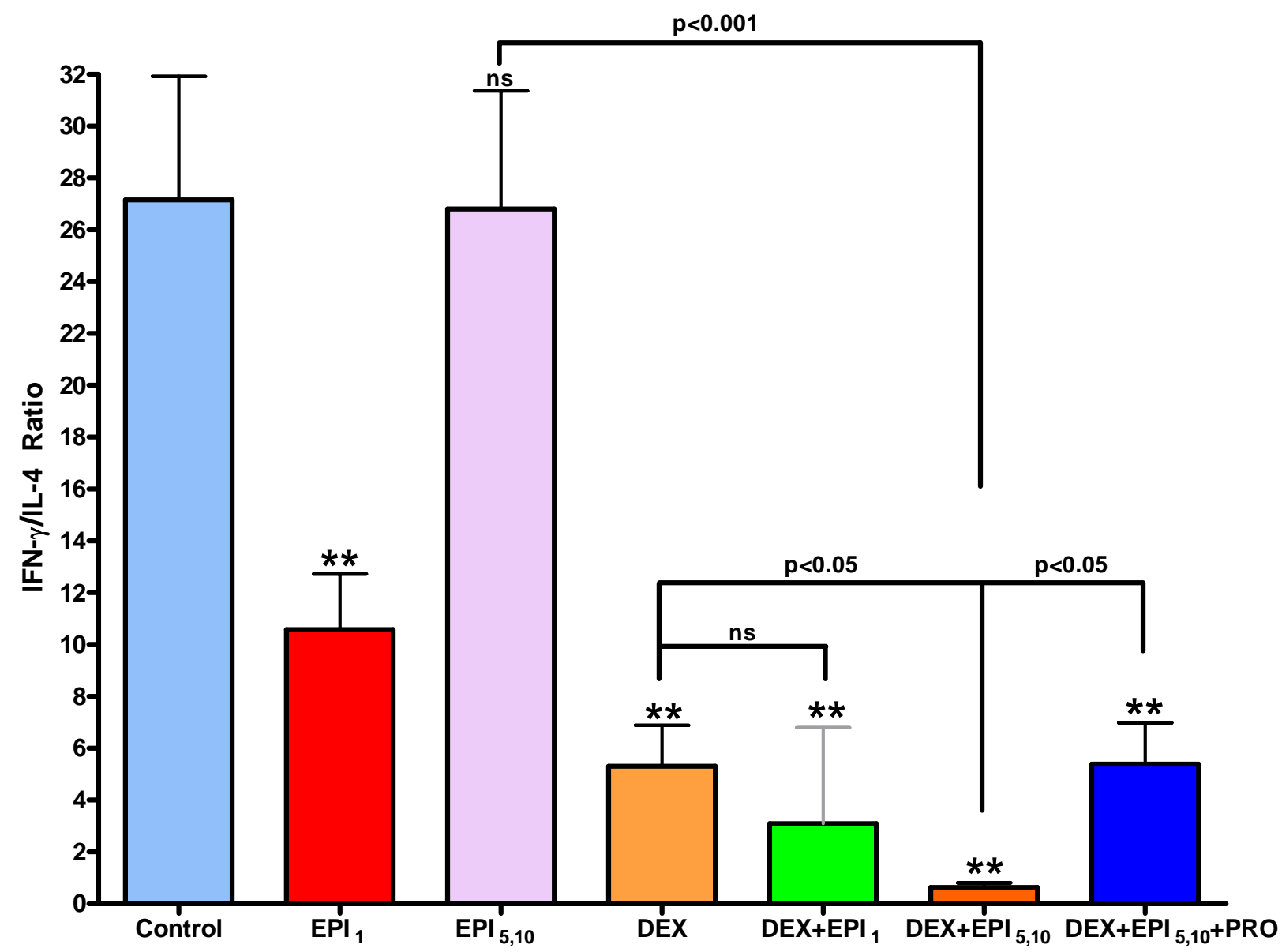

Figure 5: The balanced production of cytokines determines the adaptive immune response. Type-1/Type-2 (IFN- $\gamma /$ IL-4) cytokine ratios were calculated from ELISA data of 11-day TT (antigen-specific) stimulated cultures. The balanced production of cytokines determines the adaptive immune response. Type-1/Type-2 (IFN- $\gamma / \mathrm{IL}-10)$ cytokine ratios were calculated from ELISA data of 11-day TT (antigen-specific) stimulated cultures. The data represent the effects of physiologic-stress doses of CT added at different time points $\left(\mathrm{EPI}_{1}, \mathrm{EPI}_{5,10}\right)$, CS (DEX), the combination of CT $+\mathrm{CS}\left(\mathrm{DEX}+\mathrm{EPI}_{1}, \mathrm{DEX}+\mathrm{EPI}_{5,10}\right)$, or CT+CS+propranolol $\left(\mathrm{DEX}+\mathrm{EPI}_{5,10}\right.$ +PRO) in human PBMC from a sample size of 14 healthy adults. Data are represented as mean \pm standard deviation. $* *=\mathbf{p}<0.001$ and represented $\mathbf{p}$ values were calculated by Tukey's Multiple Comparisons test. 